\title{
Categorization reaction time, category structure, and category size in semantic memory using artificial categories
}

\author{
PAUL J. CASEY \\ Riverina College of Advanced Education, Wagga Wagga, New South Wales 2650, Australia
}

and

\author{
RICHARD A. HEATH \\ University of Newcastle, New South Wales 2308, Australia
}

\begin{abstract}
This study examined the effects of category structure and category size on categorization reaction time, artificial categories being used to obtain greater experimental control than is usually found in semantic memory research. Four artificial categories varying in structure (hierarchical and nonhierarchical) and size (8 or 16 instances) were introduced to 20 adolescent subjects over a 12-week period by means of stories, exercises, and discussions. Significantly longer categorization reaction times were required for instances from the hierarchical categories, but no set size effect was found. The application of clustering and multidimensional scaling procedures to subjects' free recall data revealed that subjects had acquired the hierarchical structures but imposed their own structures on the other categories. These findings were interpreted within a spreading activation framework.
\end{abstract}

Research in semantic memory has relied heavily on the natural memories of subjects, and relatively few studies have attempted to use artificial information. The few include Smith, Haviland, Buckley, and Sack (1972), who used learned artificial facts in order to examine the confounding in the Collins and Quillian (1969) memory organization research between noun-property frequency and number of hypothesized deductive steps, and Potts (1976), who examined the ability of subjects to correctly accept or reject assertions based on previously learned artificial logical relationships. Recently, Murphy and Smith (1982) used artificial category names in order to control for both the length and familiarity of natural category names. None of these experiments, however, has examined the spontaneous generation of a semantic memory structure for unfamiliar stimulus material over a lengthy training period.

Awareness of problems associated with the use of natural memories has led to both caution and debate over the control of relevant variables. Landauer and Meyer (1972) discussed the problems encountered in determining word frequency and relative sizes of semantic categories, as well as the confounding introduced by

This paper is based on an MA thesis submitted to the University of Newcastle by the first author and supervised by the second author. We wish to thank the subjects for their generous participation in the project for a period of almost 12 months. words with distinctive phonetic markers (e.g., "ology") and categories with an unusually large proportion of short or long words. Anderson and Bower (1973) doubted if factors such as word frequency, conjoint propositional frequency, recency, and concreteness could be adequately controlled.

The debate over measurement of category size illustrates the seemingly intractable control problem using natural memories. Methods of measuring category size can be summarized as being either analytic or subject-based (Wilkins, 1979). The analytic methods are illustrated by the use of nested pairs of categories, as in Landauer and Freedman (1968), and the counting of exemplars in some compendium of English words, such as Thorndike and Lorge (1944). The nesting technique introduces the confounding variable of greater abstractness of the larger categories. The counting of exemplars assumes that the listings in a popular thesaurus are exhaustive. However, thesauri may list more common words while omitting rare but easily classifiable words. If the frequency distributions along a "commonness" dimension were similar for all categories, then a reasonable measure of category size might be the number of common words in the category. However, large categories such as "plant" may contain many uncommon words, whereas relatively small categories such as "days of week" may contain all common words. Wilkins (1979) has warned also that analytic measures do not take into account the manner in which a subject may 
interpret the category name, for example, "mammal" possibly being confused with "animal."

The subject-based methods include subjects' production of exemplars (Battig \& Montague, 1969) and subjects' ratings of category size (Morris, 1977). Morris has objected to the method of production of exemplars in a limited time on the grounds of problems introduced by differing item accessibility. It can be added that in the semantic memory categorization task, the subject is required to recognize rather than produce instances of a given category. The number of instances produced by a subject for a given category would not necessarily be monotonically related to the number of instances that the subject could recognize. For example, a subject might produce more names of "months" than "birds" even though the latter is the larger category. The method of subjects' ratings of category size (Morris, 1977) has been criticized by Wilkins (1979) on the grounds that the experimenter does not know by what criteria category size estimates are made. This ignorance could lead to a circular operational definition of category size with no external validation, categories with a high rating being large and large categories being those with a high rating.

The selection of particular categories can also provide difficulties in avoiding overlap between categories. For instance, Loftus (1975) drew attention to Rosch's (1975a) categories, in which members of the category "vehicle" such as "car" and "skates" could also be members of the category "toy."

Several factors have been proposed as the critical influence on reaction time (RT) in categorization experiments. For example, Collins and Quillian (1969, 1970) have proposed inferential distance as the critical factor, with nesting and semantic relatedness being relevant. Landauer and Freedman (1968), Landauer and Meyer (1972), and others have pointed out that increasing inferential distance is effective in increasing the set size. Conrad (1972) has argued that conjoint frequency of category and instance is the significant factor. However, the role of such factors as category size, nesting, and conjoint frequency is difficult to assess using natural memories because of inherent control problems.

The purpose of this study was to examine the effects of particular category structures and category sizes on categorization RT using artificial categories. A set of artificial categories was developed and introduced to subjects over a period of several months. Frequent involvement with these concepts ensured that subjects did not simply have a list of instances committed to memory, but rather, a familiar set of concepts, each of which would be associated with a particular image and verbal description.

The use of artificial categories would introduce special difficulties, since the terms and their meaning would be assimilated into an existing memory framework. Hence, the experience of subjects would play some role in the eventual meaning of a concept. On the other hand, set size and printed frequency could be controlled precisely, and printed conjoint frequency, although difficult to control in prose, could be controlled by way of written exercises. The structure of the concept could be controlled, and category familiarity would vary little in newly learned, artificial categories. Findings from Joelson and Herrmann (1978) indicated that major factors that affect the use and comprehension of categories are category size, category-label printed frequency, semantic complexity, and category familiarity. This study aimed to control these factors as far as possible.

Four categories were developed, each containing either 8 or 16 instances, and each being either hierarchically organized or unorganized. The training period of 12 weeks distinguished this study from that of Potts (1976) and Smith et al. (1972), since their subjects studied the given information for only a few mintues. The subjective group structures for each category were examined by using a technique for inducing the organization of information in memory based on regularities in free recall, as proposed by Friendly (1977). The Friendly (1977) technique uses as the measure of similarity the average number of instances between each possible pair of instances in free recall lists of the category. The similarity matrices derived from subjects' free recall of the instances of each category were analyzed for structure by means of multidimensional scaling and clustering procedures.

The posttraining experimental task, which required subjects to decide whether or not individually presented instances were members of a previously presented superset, allowed the testing of several hypotheses. One hypothesis, based on much of the literature, was that the duration of categorization RT would vary directly with the size of the target category (Landauer \& Freedman, 1968; Landauer \& Meyer, 1972; Meyer, 1970; Wilkins, 1971). A competing hypothesis, based on Collins and Quillian (1970), was for an effect due to hierarchical structure but not set size. If the artificial categories were to behave similarly to natural categories, then negative RTs would be longer than positive RTs (e.g., Wilkins, 1971), and the negative categorization of well learned instances would take longer than the negative categorization of novel instances (e.g., Smith, Shoben, \& Rips, 1974)

\section{METHOD}

\section{Development of Artificial Categories}

Construction of artificial categories. Sixty-eight consonantvowel-consonant (CVC) trigrams were selected from Archer (1960) within the 45-70 range of a 1-100 scale of meaningfulness. CVCs with " $Y$ " as the vowel, ending in " $C$," " $H$," " $Q$," or "W," which would cause pronunciation difficulties, or having a sex-related rating difference were not chosen. Subjects were advised of the pronunciation of all words.

CVCs were randomly assigned as category names, subcate- 
gory names, and instances. Four CVCs were reserved as "unseens." With one exception, no two instances in a given category were allowed to start with the same consonant. While an effort was made to avoid having words that rhymed with others, the limited number of otherwise suitable CVCs made some rhyming unavoidable. The category distribution of CVCs is given in Table 1.

Imposition of attributes. In order to make the artificial categories as similar as possible to natural categories, care was taken in the selection of the defining attributes of the categories, subcategories, and instances. The common attribute of all the categories was that they were able to move in some fashion, the defining attribute of each set being its unique mode of movement. Each subcategory of the structured sets was given a defining set of attributes, and all instances of all sets were given two defining attributes. A brief description of each of the categories and subcategories is given in Table 2 .

\section{Training of Subjects}

Subjects. The subjects were 30 volunteers from Years 6,7 , and 8 from local schools. There were 25 females and 5 males. During training, seven subjects left the program for varying personal reasons. Three failed a pretest. The age range of the remaining 20 subjects at the commencement of the program was from 11 years 2 months to 14 years 1 month, the mean age being 12 years 6 months. These 20 subjects were successful on a series of Piagetian classification tasks, designed by Taufer'Langke (cited in Sprinthall \& Sprinthall, 1977), which tested ability to manipulate class inclusion relations.

Training procedure. Preliminary oral instructions advised subjects that they were to learn as much as possible about life on an imaginary planet by way of stories, exercises, and discussion spread over a 12 -week period. No specific details were given as to the nature of the subsequent experimental task. Two or three story-exercise sets were distributed each week, up to a total of 30 sets. The subjects were requested to space their study evenly over the week.

The order of study of particular categories over the 12-week training period was as follows. All subjects, because of organizational constraints, began their study with JEP(H-16), then followed TOX $(\mathrm{N}-8), \operatorname{DIB}(\mathrm{N}-16)$, and LUT(H-8). This initial phase required 6 weeks. Next, over a 3-week period, subjects read stories and performed exercises in which the categories and instances were encountered in a pseudorandom order. In the final 3 weeks, to counteract an order-of-learning effect, subjects participated in 15 tutorial sessions, during which they individu-

Table 1

Allocation of CVCs to Categories by Set Size and Structure

\begin{tabular}{|c|c|c|c|c|c|c|c|c|c|}
\hline \multicolumn{5}{|c|}{ Large Set (16) } & \multicolumn{5}{|c|}{ Small Set (8) } \\
\hline $\begin{array}{c}\text { Nonhier- } \\
\text { archical (N) } \\
\text { DIB(N-16)* }\end{array}$ & \multicolumn{4}{|c|}{$\begin{array}{c}\text { Hierarchical }(H) \\
\text { JEP(H-16) }\end{array}$} & $\begin{array}{c}\text { Nonhier- } \\
\text { archical (N) } \\
\text { TOX }(\mathrm{N}-8)\end{array}$ & \multicolumn{4}{|c|}{$\begin{array}{l}\text { Hierarchical }(\mathrm{H}) \\
\text { LUT(H-8) }\end{array}$} \\
\hline PES & \multicolumn{2}{|c|}{ BEM } & \multicolumn{2}{|c|}{ PAB } & $\mathrm{BOZ}$ & \multicolumn{2}{|c|}{ GID } & \multicolumn{2}{|c|}{$\mathrm{KOV}$} \\
\hline TIG & VAP & FIS & HET & $\underline{\mathrm{MEZ}}$ & DEV & SEV & $\underline{\mathrm{NAL}}$ & YID & ZEP \\
\hline GOM & $\overline{\mathrm{KAF}}$ & $\overline{\mathrm{GUF}}$ & $\overline{\mathrm{BEF}}$ & $\overline{\mathrm{FAV}}$ & SAF & $\mathrm{ZOM}$ & TUD & $\overline{\text { WOL }}$ & JOD \\
\hline VUL & MOG & HIN & TEP & PUM & $\mathrm{HAB}$ & $\mathrm{DEG}$ & KUL & $\mathrm{GAZ}$ & MAV \\
\hline BAX & ZIT & NOM & SIZ & WEP & NUP & & & & \\
\hline FEZ & $\mathrm{DOF}$ & VOD & LIF & VIG & JIT & & & & \\
\hline JUM & & & & & FOL & & & & \\
\hline MIP & & & & & PIV & & & & \\
\hline SOF & & & & & & & & & \\
\hline NEP & & & & & & & & & \\
\hline YAL & & & & & & & & & \\
\hline HUK & & & & & & & & & \\
\hline ZAR & & & & & & & & & \\
\hline KEM & & & & & & & & & \\
\hline WUD & & & & & & & & & \\
\hline LEB & & & & & & & & & \\
\hline
\end{tabular}

*Future references to the category titles, DIB, JEP, TOX, and LUT, will include a memory aid indicating the structure and size of the category. Thus, DIB(N-16) indicates that DIB is a nonhierarchically organized category containing 16 instances.

Table 2

Defining Attributes of Categories and Subcategories

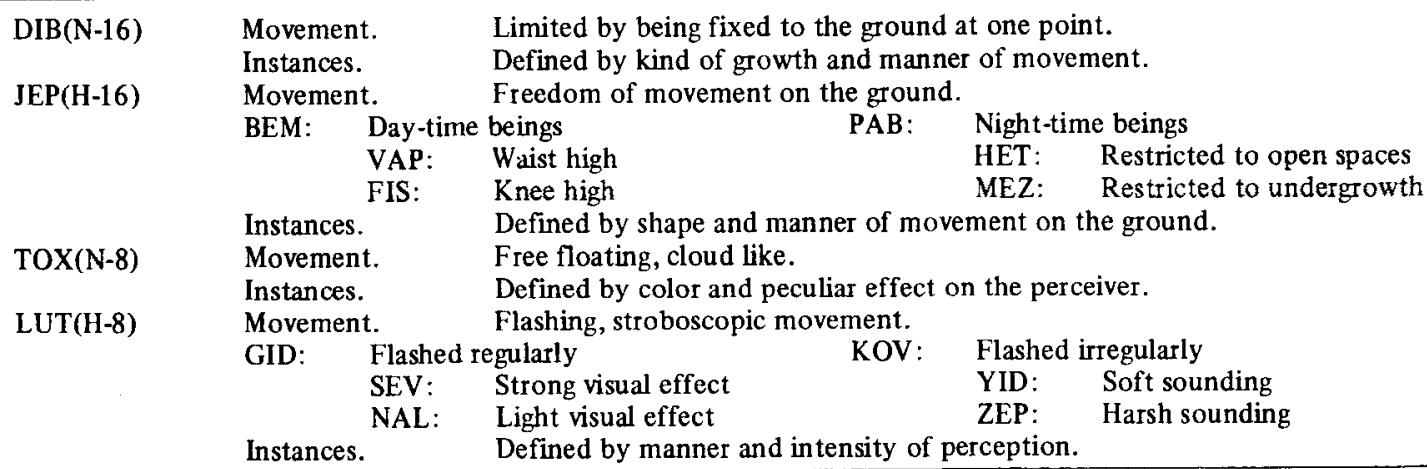


ally reviewed the categories in different, pseudorandom orders. The exercises for these sessions involved recalling, recognizing, describing, categorizing, imagining, discussing, story telling, and role playing, the aim being to make the CVCs a meaningful part of the subjects' vocabulary.

The mean number of formal study sessions per subject over the 12-week training period was approximately 35. Regular attainment tests were held, which included the recall of instances, and when appropriate, category subset names. At the conclusion of training, 20 subjects successfully performed a written descriptive recall of all the instances grouped within their appropriate categories.

Design of stories. The stories consisted of an introduction to "Life on Planet VIZ," a fictitious planet visited by two Year 7 students as part of their science course. The two visitors reported what they learned about life on VIZ in 18 diary segments. Each diary report was followed by questions and excrcises for the subjects. Twelve of the 30 distributed work sheets contained only review exercises. Sample diary reports are given in the appendix.

Care was exercised in balancing the printed frequencies of categories and instances. The category names, DIB(N-16), JEP(H-16), TOX(N-8), and LUT(H-8), were printed approximately 60 times each. The major subset names of the two hierarchical categories, JEP(H-16) and LUT(H-8), occurred approximately 30 times, and the minor subset names for these categories were printed 25 times. Instances of the four categories were printed seven to eight times each.

The conjoint frequencies of superordinate categories and their associated instances [e.g., PES-DIB(N-16), KAF-JEP(H-16), BOZ-TOX $(\mathrm{N}-8)$, and ZOM-LUT(H-8)] were balanced across categories (in the manner described further below). However, the conjoint frequencies of instances and different-level superordinate categories in the hierarchical categories [e.g., KAF-VAP, KAF-BEM, KAF-JEP(H-16)] could not be balanced, thus preventing a controlled replication of the Collins and Quillian (1969) research on the relationship between RT and inferential distance within a hierarchy. The failure to attempt such a conjoint balance rested on the intention to compare categories varying both in structure and in size. Control of printed frequency was a basic methodological requirement. However, if conjoint frequency was balanced, not only across categories, but also within the varying levels of the hierarchical categories [e.g., balancing PES-DIB(N-16) and KAF-VAP, KAF-BEM, KAF-JEP(H-16)|, then the instances of the hierarchical categories would occur three times as frequently as instances of the nonhierarchical categories. The framework of the stories prohibited the tripling of the nonhierarchical instances without also increasing the conjoint frequency of these instances and their superordinate categories [e.g., PES-DIB(N-16), BOZ-TOX(N-8)|, thus disturbing the balance of conjoint frequencies across the four categories. Hence, an effect of nesting instances within the JEP(H-16) and LUT(H-8) categories was examined by comparing categorization RTs of instances for the major superordinate categories of these sets [viz., JEP(H-16) and LUT(H-8)] with categorization RTs of instances of the nonhierarchical sets. The presence of subjective hierarchical structures for JF: $\mathrm{P}(\mathrm{H}-16)$ and LUT $(\mathrm{H}-8)$ could be examined by means of clustering and multidimensional scaling procedures, as described in the results section.

Balance of conjoint frequency was attempted by having subjects read instances and associate them with appropriate major superordinate categories. Approximately 20 of the weekly review exercises required subjects to describe each instance in each category, confirm or deny instances as members of nominated categories, or categorize lists of instances. Hierarchical category exercises also had subjects group instances into the varying subsets. Emphasis was given to the irrelevance of order of instances within categories and. for the hierarchical sets, with in subordinate categories.

\section{Categorization Task}

Sets of $35-\mathrm{mm}$ slides of the 48 instances and the unseen instances BIM. DUT, MUZ, and POV were prepared. Twelve training slides with categories and instances taken from "animal" and "building" categories were also composed. A different pseudorandom order of the 32 slides was prepared for each subject. Four positive instances were randomly selected from each category. For the negative instances, one instance not used as a positive instance was selected from each of the other categories, and the fourth was one of the four unseen CVCs listed above. In the final ordering, no more than three positive or three negative instances were allowed to occur successively. The same category was not tested more than twice in succession. A runs test found that no ordering for any subject deviated significantly from a random ordering.

Subjects were instructed that on each trial, one of the superset category names, DIB $(\mathrm{N}-16)$, JEP $(\mathrm{H}-16)$, TOX $(\mathrm{N}-8)$, or LUT(H-8), would be given orally. Approximately $2 \mathrm{sec}$ later, a CVC would be presented on the screen. The subjects' task was to indicate the categorization decision by depressing one of two response buttons that indicated the responses "Yes" and "No."

\section{Apparatus}

Slides of the instances on $35 \cdot \mathrm{mm}$ film were presented with the aid of a tachistoscopic shutter attached to a Kodak Carousel projector. A millisecond timer, activated by the release of the tachistoscopic shutter and stopped by the depression of one of the two hand-held response buttons, was used to measure RT.

\section{Design and Procedure}

A 2 by 2 by 2 randomized-blocks design was employed, with each subject corresponding to a block. Factors were set size ( 8 and 16 instances), set structure (hierarchical and nonhierarchical), and response type (positive and negative). The subjects, who were right-handed, were seated $2.5 \mathrm{~m}$ from the screen. They were directed to indicate if the instance belonged to the orally presented category by depressing one of the two response buttons. The mapping of the responses on to the right and left buttons was varied between subjects to control for handedness effects. The instructions, which emphasized accuracy, also required subjects to respond as quickly as possible.

The experimental trials were preceded by 12 practice slides. Following the RT task, subjects were required to recall the instances in each category.

\section{RESULTS}

The major finding was that category structure but not category size significantly affected categorization RT, longer RTs being required to categorize instances for hierarchical categories than for nonhierarchical categories. Means and standard errors of mean RTs are given in Table 3. As explained in the method section, methodological constraints prevented the examination of distance effects within the hierarchical categories.

The subjects committed only nine errors $(1.4 \%)$, which were unsystematically distributed across the eight conditions. Consequently, error RTs were omitted from the data analysis. An analysis of variance for a 2 by 2 by 2 randomized-blocks design with mixed effects produced the following results. The mean RT for the categorization of instances from the hierarchically organized categories, JEP(H-16) and LUT(H-8), was significantly longer than RT for the nonhierarchically organized categories. DIB(N-16) and TOX $(\mathrm{N}-8)[\mathrm{F}(1,19)$ $=6.04 . \mathrm{MSe}=.232, p<.025]$. Mean RT for negative 
Table 3

Means and Standard Errors (SE) of Means of Mean Categorization RTs in Seconds

\begin{tabular}{|c|c|c|c|c|c|c|c|c|}
\hline & \multicolumn{4}{|c|}{ Positive Response } & \multicolumn{4}{|c|}{ Negative Response } \\
\hline & \multicolumn{2}{|c|}{ Large Set } & \multicolumn{2}{|c|}{ Small Set } & \multicolumn{2}{|c|}{ Large Set } & \multicolumn{2}{|c|}{ Small Set } \\
\hline & $\operatorname{DIB}(\mathrm{N}-16)$ & JEP(H-16) & TOX $(N-8)$ & $\operatorname{LUT}(\mathrm{H}-8)$ & DIB(N-16) & JEP(H-16) & TOX(N-8) & LUT(H-8) \\
\hline $\begin{array}{l}\text { Mean RT } \\
\text { SE }\end{array}$ & $\begin{array}{r}1.754 \\
.184\end{array}$ & $\begin{array}{r}2.037 \\
.189\end{array}$ & $\begin{array}{r}1.581 \\
.116\end{array}$ & $\begin{array}{r}2.002 \\
.215\end{array}$ & $\begin{array}{r}2.302 \\
.222\end{array}$ & $\begin{array}{r}2.155 \\
.200\end{array}$ & $\begin{array}{r}1.923 \\
.162\end{array}$ & $\begin{array}{r}2.115 \\
.205\end{array}$ \\
\hline
\end{tabular}

responses was significantly longer than mean RT for positive responses $[\mathrm{F}(1,19)=6.74, \mathrm{MSe}=.467, \mathrm{p}<.025]$. There was no set size effect $[F(1,19)=2.21]$. The Response Type by Category Structure interaction approached significance $[F(1,19)=4.02]$. The Category Size by Category Structure interaction was nonsignificant $[F(1,19)=1.74]$, and the remaining two interactions were also nonsignificant $[\mathrm{F}(1,19)<1]$.

The expectation that well learned instances would take longer to categorize negatively than unseen instances was verified. Group mean RTs for negative categorizations are given in Table 4. A randomized-blocks design analysis of averaged negative categorization RTs grouped according to the source of a negative [i.e., from DIB(N-16), TOX(N-8), JEP(H-16), LUT(N-8) or an unseen category] yielded $\mathrm{F}(4,76)=14.90(\mathrm{MSe}=.313$, $\mathrm{p}<.001)$. A Duncan's multiple-range test showed that the only significant differences were between instances from the unseen category and all other categories $(\mathrm{p}<.001)$.

The application of multidimensional scaling and clustering techniques to the four similarity matrices derived from the subjects' written free recall of the instances of each of the categories (cf. Friendly, 1977) showed that the subjective group structures for the JEP(H-16) and LUT(H-8) categories were the same hierarchical structures imposed by the experimenters. Further, a degree of structure had been imposed by the group on the DIB $(\mathrm{N}-16)$ and $\operatorname{TOX}(\mathrm{N}-8)$ categories. The multidimensional scaling technique employed was that of Kruskal (1964a, 1964b), available as the computer program M-D-SCAL (Version 5M, Kruskal, 1976). Clustering was achieved by the complete-link method, using the BMDP1M program (Dixon \& Brown, 1979).

The one- and two-dimensional stress values resulting from the M-D-SCAL analysis of the similarity matrices
Table 4

Group Mean RTs in Seconds for Negative Categorizations by Source of Negative

\begin{tabular}{cccccc} 
& \multicolumn{5}{c}{ Source of Negative } \\
\cline { 2 - 6 } & $\begin{array}{c}\text { DIB } \\
\text { (N-16) }\end{array}$ & $\begin{array}{c}\text { JEP } \\
(\mathrm{H}-16)\end{array}$ & $\begin{array}{c}\text { TOX } \\
(\mathrm{N}-8)\end{array}$ & $\begin{array}{c}\text { LUT } \\
(\mathrm{H}-8)\end{array}$ & UNSEEN \\
\hline Mean RT & 2.235 & 2.452 & 2.388 & 2.501 & 1.338 \\
\hline
\end{tabular}

are presented in Table 5. The distribution of the stress statistic developed by Levine (1978) was used to determine whether or not the sets of similarity data had other than random structure. Average stress and standard deviation for a given number of points and dimensions were taken from Table 1 of Levine (1978, p. 309). The $\mathrm{z}$ scores and associated probabilities, given in Table 5, showed that all categories had a structure deviating significantly from chance.

The cluster analyses of the similarity data from the JEP(H-16) and LUT(H-8) categories revealed that subjects had either acquired or imposed nearly perfect hierarchical structures. The tree produced for the LUT category is shown in Figure 1. The superimposition in Figure 2 of the JEP(H-16) clustering solution onto the JEP(H-16) two-dimensional M-D-SCAL solution shows the groupings for JEP(H-16), as in Table 1, and the strong tendency toward unidimensionality.

While the hierarchical categories appeared to have subjectively equivalent structures, DIB(N-16) and TOX $(\mathrm{N}-8)$ differed in structure from the hierarchical categories and from each other. The cluster analysis for TOX(N-8), depicted in Figure 3, suggested relatively high levels of similarity between the pairs of instances DEV-BOZ, JIT-NUP, and PIV-FOL. The cluster solution for DIB(N-16) is shown in Figure 4 superimposed on the two-dimensional M-D-SCAL solution.

Table 5

Stress Percentages and Levine (1978) Derived z Scores for One- and Two-Dimensional M-D-SCAL Configurations of the Artificial Categories

\begin{tabular}{|c|c|c|c|c|c|c|c|c|}
\hline \multirow{3}{*}{$\begin{array}{l}\text { Number of } \\
\text { Dimensions }\end{array}$} & \multicolumn{8}{|c|}{ Category } \\
\hline & \multicolumn{2}{|c|}{ JEP(H-16) } & \multicolumn{2}{|c|}{ LUT(H-8) } & \multicolumn{2}{|c|}{ DIB(N-16) } & \multicolumn{2}{|c|}{ TOX $(\mathrm{N}-8)$} \\
\hline & Stress & $\mathrm{z}$ & Stress & $z$ & Stress & $z$ & Stress & z \\
\hline One & 7.15 & $21.29 \dagger$ & 4.14 & $6.63 \dagger$ & 74.77 & $2.35^{*}$ & $\begin{array}{r}18.38 \\
3.49\end{array}$ & $\begin{array}{l}5.06 \dagger \\
3.92 \dagger\end{array}$ \\
\hline
\end{tabular}

${ }^{*} p<.01 . \quad t p<.0001$. 


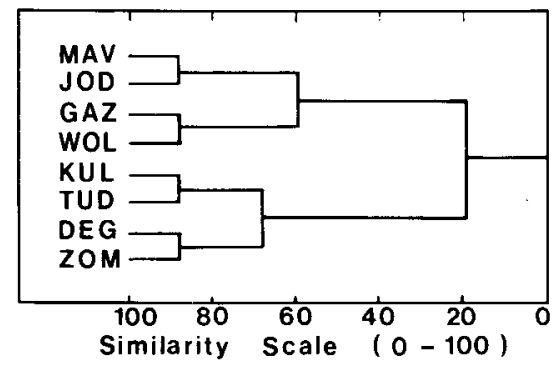

Figure 1. Tree produced from the similarity matrix for the LUT(H-8) category (scaled 0-100) from clustering by the complete linkage method.

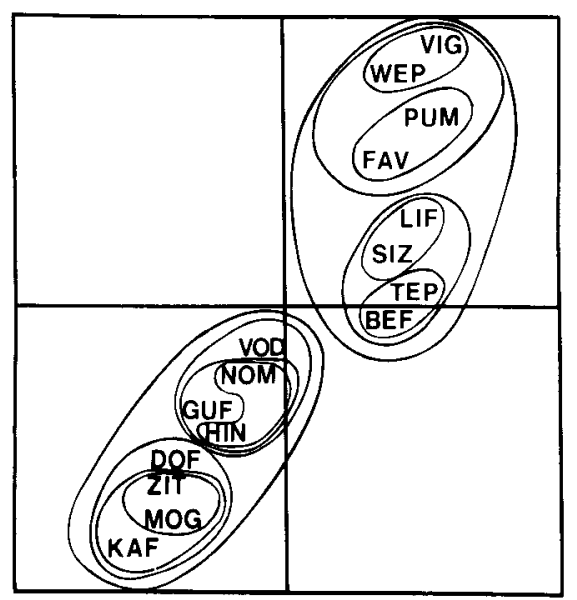

Figure 2. Two-dimensional M-D-SCAL representation of the JEP(H-16) category, with loops indicating clusters from the BMDP1 M cluster analysis.

\section{DISCUSSION}

The results supported the Collins and Quillian (1970) finding that categorization RT is a function of hierarchical structuring but not of category size. The hypotheses of longer RTs for negative categorizations over positive categorizations and for negative categorization of well learned instances over novel instances were supported. The M-D-SCAL and clustering procedures showed that the experimenter-imposed hierarchical structures were also the subjective structures. Further, as shown by Tulving (1962), subjects imposed their own organization on the "unrelated" instances of DIB(N-16) and TOX(N-8).

The effect of category structure but not category size is contrary to a category search model (e.g., Landauer $\&$ Meyer, 1972). The notion of search may not be appropriate when instances are both well learned and clearly defined in number, even though such a notion may be appropriate in the case of small, newly learned categories defined by only an enumeration of instances (e.g., Pollack, 1963; Sternberg, 1966).

The major findings cannot be explained by a feature comparison model, such as that of Smith et al. (1974),

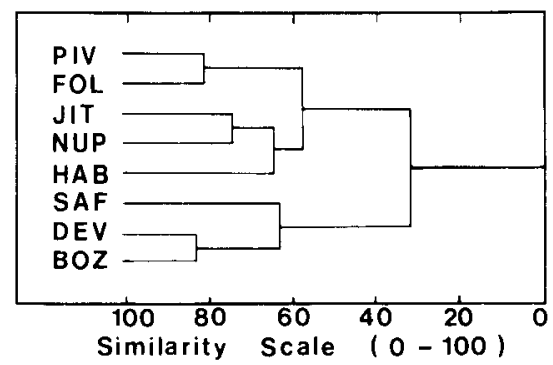

Figure 3. Tree produced from the similarity matrix for the TOX (N-8) category (scaled $0-100$ ) from clustering by the complete linkage method.

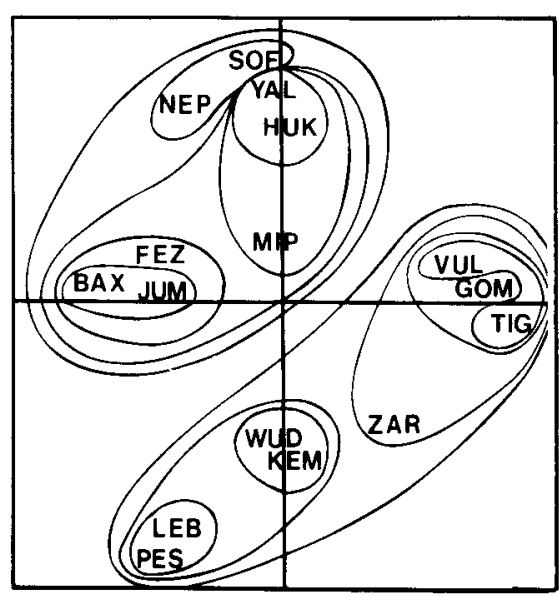

Figure 4. Two-dimensional M-D-SCAL representation of the DIB(N-16) category, with loops indicating clusters from the BMDP1M cluster analysis.

since the predictions from such a model for category size effects are not clear. Such predictions rest on the relative similarity between instance and category, the concept of similarity being related to defining and characteristic features. In this experiment, no attempt was made to measure similarity, assuming such a concept differs from conjoint frequency. Moreover, Collins and Loftus (1975) have pointed out that the distinction between defining and characteristic features in real categories cannot be maintained.

While Hollan (1975) has demonstrated that settheoretic models, such as that of Smith et al. (1974), are equivalent to network models, different models appear to give rise to different ways of interpreting the data (Gellatly \& Gregg, 1977). Spreading-activation theory (e.g., Anderson, 1976) can be modified to account for the effect of hierarchical structure and the absence of a category size effect. Spreading-activation theory, as proposed by Collins and Loftus (1975), has been considered lacking in empirical falsifiability (Anderson, 1976; Rosch, 1975b), and the more testable ACT model of Anderson (1976) is also worrisome to its author in regard to its degree of empirical testability (p. 531). Given this concern about spreading-activation 
theories, the following exposition rests on the general notion of activation emanating through the conceptual network from activated nodes, combined with a hypothesized decision making process.

In the present experiment, there were four well learned categories. After a target category was presented [e.g., JEP(H-16), DIB(N-16)], 2 sec elapsed before an instance was presented, such time being sufficient for all instances of the given category to be activated, even though activation may have been slower for the larger categories. Moreover, because the conjoint frequencies of major superordinate categories and instances were balanced both across and within categories, activation downward in any category should have resulted in all instances being equally well primed. Here lies an important difference from natural categories. In natural categories, it is arguable that instances that have a higher conjoint frequency are more highly primed. In larger categories, there may be many instances that are very weakly primed. However, with artificial categories, priming allowed activation of all instances in roughly equal amounts.

The presentation of a positive instance following priming produced different consequences for hierarchical categories than for nonhierarchical categories. In the former, if a positive instance of JEP(H-16), such as $\mathrm{KAF}$, was presented, activation would spread upward in parallel to all three superordinates, VAP, BEM, and JEP, since subjects had learned the categorizations KAF-VAP, KAF-BEM, and KAF-JEP (even though the conjoint frequencies of such pairs were unable to be balanced). The decision process affirming "A KAF is a JEP" would be slowed down by interference from the associations KAF-VAP and KAF-BEM. However, if a positive instance of a nonhierarchical category was presented [e.g., PES from DIB(N-16)], only one superordinate (viz., DIB) would be activated. The upward activation required to confirm instances as being from nonhierarchical categories would receive less interference than in the case of instances from hierarchical categories, thus resulting in shorter categorization RTs for instances from nonhierarchical categories.

In summary, priming produced approximately equal activation of all instances of the given category. Subjects, on presentation of an instance, sought confirmatory evidence by checking the link to the superordinate category. This confirmation process was slower in hierarchical structures because of interference from instances being linked with three superordinates. The category size effect in many earlier experiments (e.g., Landauer \& Freedman, 1968; Wilkins, 1971) was attributed in part to differential activation of instances, which was circumvented in this study by the control of conjoint frequency. It may be that a category size effect would be found if categories were not primed. Moreover, the RT data suggest that there may be a category size effect for the nonhierarchical categories that is masked by variability in the data.
The usual findings of longer categorization RTs for negative categorizations than for positive categorizations and shorter negative categorizations for novel instances than for semantically closer instances can also be related to spreading activation and a subsequent decision process. It is worth noting that negative instances always came from another category (only superordinate categories were tested), were used only once, and had never served as a positive instance.

When a category was presented, since all four categories came from the imaginary planet VIZ, a weak priming effect occurred for the instances of the categories not being considered. If the negative instance presented was one of the four unseen instances, no further activation occurred and such an instance was immediately categorized negatively. However, a weakly primed, negative instance required checking by tracing a path to its superordinate node. Moreover, the superordinate category might be a hierarchical category (as was the case for $50 \%$ of negative instances for the nonhierarchical categories but only for $25 \%$ of the hierarchical categories), which would slow down the checking process. Thus, the first step was to check links for a positive categorization. If such links were weak, the next step was to generate the superordinate category of the given instance and falsify that this was the target category. Thus, a tendency to seek confirmatory evidence resulted in an additional step for negative categorizations, and hence, longer RTs.

Caution clearly must be exercised in generalizing findings from artificial to natural categories. For example, natural categories may lack clearly definable features. Moreover, the use of hierarchical categories in which the highest conjoint frequency is between instances and the major superordinate may be unnatural. However, given the correlational nature of much semantic memory research (Anderson \& Reder, 1974) and the control problems discussed in the introduction, artificial categories can be employed quite profitably in semantic memory research. The results of this study have shown that, not only can well known findings be replicated using artificial categories, but also some light may be cast on the less tractable problems of semantic memory such as the effects of category size and structure.

\section{REFERENCES}

Anderson, J. R. Language, memory and thought. New York: Wiley, 1976.

Anderson, J. R., \& Bower, G. H. Human associative memory. New York: Wiley, 1973.

Anderson, J. R., \& Reder, L. Negative judgments in and about semantic memory. Journal of Verbal Learning and Verbal Behavior, 1974, 13, 664-681.

ARCHER, E. J. A re-evaluation of the meaningfulness of all possible CVC trigrams. Psychological Monographs, 1960, 74, 1-23.

Battig, W. F., \& Montague, W. E. Category norms for verbal items in 56 categories: A replication and extension of the 
Connecticut category norms. Journal of Experimental Psychology Monograph, 1969, 80, 1-46.

Collins, A. M., \& LofTus, E. A spreading-activation theory of semantic processing. Psychological Review, 1975, 82, 407-428.

Collins, A. M., \& Quillian, M. R. Retrieval time from semantic memory. Journal of Verbal Learning and Verbal Behavior, $1969,8,240-247$.

Collins, A. M., \& Quillian, M. R. Does category size affect categorization time? Journal of Verbal Learning and Verbal Behavior, 1970, 9, 432-438.

Conrad, C. Cognitive economy in semantic memory. Journal of Experimental Psychology, 1972, 92, 149-154.

Dixon, W. J., \& Brown, M. B. (Eds.). BMDP-79 biomedical computer programs $P$-series. Berkeley: University of California Press, 1979.

Friendiy, M. L. In search of the M-gram: The structure of organization in free recall. Cognitive Psychology, 1977, 9, 188-249.

Gellatly, A. R. H., \& GregG, V. H. Intercategory distance and categorization times: Effects of negative-probe relatedness. Journal of Verbal Learning and Verbal Behavior, 1977, 16, 505-518.

Hollan, J. D. Features and semantic memory: Set-theoretic or network model? Psychological Review, 1975, 82, 154-155.

Joelson, J. M., \& Herrmann, D. J. Properties of categories in semantic memory. American Journal of Psychology, 1978, 91, 101-114.

KrUSKal, J. B. Multidimensional scaling by optimizing goodness of fit to a nonmetric hypothesis. Psychometrika, 1964, 29, 1-27. (a)

Kruskal, J. B. Nonmetric multidimensional scaling: A numerical method. Psychometrika, 1964, 29, 115-129. (b)

Kruskal, J. B. M-D-SCAL. Murray Hill, N.J: Bell Telephone Laboratories, 1976.

LANDAUER, T. K., \& FreEdman, J. L. Information retrieval from long-term memory: Category size and recognition time. Journal of Verbal Learning and Verbal Behavior, 1968, 7, 291-295.

Landauer, T. K., \& Meyer, D. E. Category size and semantic memory retrieval. Journal of Verbal Learning and Verbal Behavior, 1972, 11, 539-549.

LEvine, D. M. A Monte Carlo study of Kruskal's variance based measure on stress. Psychometrika, 1978, 43, 307-315.

LoFTUs, E. F. Spreading activation within semantic categories: Comments on Rosch's "Cognitive representations of semantic categories." Journal of Experimental Psychology: General, 1975, 104, 234-240.

MEYER, D. E. On the representation and retrieval of stored semantic information. Cognitive Psychology, 1970, 1, 242-299.

Morris, P. E. A criticism of Wilkins' (1971) measure of category size and its implication for the Smith, Shoben and Rips (1974) model of semantic memory. British Journal of Psychology, $1977,68,351-352$.

Murphy, G. L., \& Sмiтh, E. E. Basic-level superiority in picture categorization. Journal of Verbal Learning and Verbal Behavior, 1982, 21, 1-20.

Pollack, I. Speed of classification of words into superordinate categories. Journal of Verbal Learning and Verbal Behavior, $1963,2,159-165$.

Potrs, G. R. Artificial logical relations and their relevance to semantic memory. Journal of Experimental Psychology: Human Learning and Memory, 1976, 2, 746-758

Rosch, E. H. Cognitive representations of semantic categories. Journal of Experimental Psychology: General, 1975, 104, 192-233. (a)

Rosch, E. H. Reply to Loftus. Journal of Experimental Psychology: General, 1975, 104, 241-243. (b)

Smith, E. E., Haviland, S. E., Buckley, P. B., \& Sack, M.
Retrieval of artificial facts from long term memory. Journal of Verbal Learning and Verbal Behavior, 1972, 11, 583-593.

Smith, E. E., Shoben, E. J., \& Rips, L. J. Structure and process in semantic memory: A featural model for semantic decisions. Psychological Review, 1974, 81, 214-241.

Sprinthall, R. C., \& Sprinthall, N. A. Educational psychology. Reading, Mass: Addison-Wesley, 1977.

Ste Rngerg, S. High-speed scanning in human memory. Science, $1966,153,652-654$.

Thonndike, E. L., \& LoRge, I. The teacher's handbook of 30,000 words. New York: Columbia University, 1944.

Tulving, E. Subjective organization in free recall of "unrelated" words. Psychological Review, 1962, 69, 344-354.

Wilkins, A. J. Conjoint frequency, category size and categorization time. Journal of Verbal Learning and Verbal Behavior, $1971,10,382-385$.

WiLkins, A. J. Sizing up categories: A reply to Morris (1977). British Journal of Psychology, 1979, 70, 132-148.

\section{APPENDIX}

Below are sample diary reports from "Life on the Planet VIZ."

Week 2. No. 2

Diary Day 2. (Jo).

Well, Pete didn't say much the first day because we were just settling in. However, we've seen so much today that I hardly know where to begin.

After some discussion, Pete and I decided we had better look at only one class of beings at a time. A new world can be very confusing. I'm confused enough at any time. Careful ordering might help me learn better. JEP, those things which moved freely but in contact with the ground, seemed an interesting looking group to begin with.

I know scientists are supposed to give Latin and Greek names to things. Animals, plants and that sort of stuff on earth are divided into phyla and genera and all that. Well, we'll keep some form of grouping. However, l've just used up all my Greek and Latin. So we'll make up our own words, as Pete has already noted. We weren't too sure how friendly the JEP might be, so we watched from some rocks near our camp. Through our binoculars we were able to see 20 or 30 objects moving about in an open space a hundred metres from where we lay. They seemed to fall into one of only two size groupings. Because we were not yet used to distances and sizes on VIZ, we could only guess at their sizes. Some seemed to be about half our height and were shaped something like a balloon but not as smooth looking. The rest looked like they would hardly come up to our knees. These varied in shape.

Pete suggested that we give each group a name. However, it seemed to me that we needed more than two new names. Pete didn't know that I had been up during the night and had noticed that there seemed to be a lot of movement around. I told Pete that $I$ had seen many things moving around clearly in the light from some of VIZ's four moons. Some seemed to move only in the open spaces and were very saucer shaped. Other objects were moving about only in the undergrowth. These seemed to have something like hairy arms, but I wasn't sure.

Some play with the letters of the alphabet produced the following words:

BEM: Day-time JEP.

PAB: Night-time JEP.

Waist-high BEM: VAP.

Knee-high BEM: IIS.

The name for the open spaces PAB: HET.

The name for the foliage loving PAB: MEZ

More on these tomorrow. 
Exercise: Please cover above and complete. Circle either True or False.

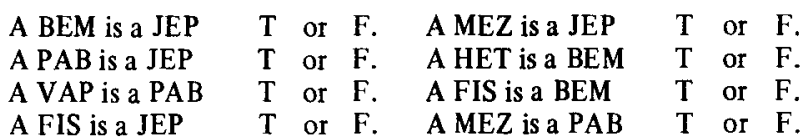

Week 9. No. 29

Diary Day 18 (Continued).

Although my day so far had been somewhat "shocking" I was determined to experiment with the LUT before "calling it a day." As evening approached I could sense that the silent GID were close by. Soon I was able to detect the fast flashing NAL and the slow flashing SEV. Then individual KUL, TUD, ZOM and DEG began to combine in a wild rhy thm of exploding light. The GID certainly could produce a powerful effect.

I took out our searchlight torch and began to signal to the GID. I tried out various flash combinations, even Morse code. When I finally managed to produce combinations of flashes which were of the same length as one of the TUD, KUL, DEG or ZOM, it seemed as if the GID moved away, perhaps mystified by some other being giving their signals.

"If I can affect the GID with regular light flashes, perhaps I might be able to contact the noisy KOV with sound combinations," I thought to myself. I put my head right back to look for the YID and searched out the corner of my eye for the ZEP, at the same time listening for distinctive sounds: the WOL siren, the GAZ humm, the JOD twang and the MAV bong. When I finally picked up the sound effects of the KOV, I turned up a cassette of my favourite group, The Wedges, to their normal, head-splitting crescendo. I thought the KOV might appreciate the music and perhaps learn something from it. Certainly the right combination of KOV sounds, GID lights and TOX colours could possibly produce a galaxy wide sensation.

The music had barely commenced when the KOV began to fade away into the distance. I moved my head appropriately, but clearly the YID and ZEP had gone. Maybe the KOV didn't have my taste in music?

Anyway, what was "taste" to these strange beings? I was becoming very aware that although we could describe these VIZ beings fairly well, we were still a long way from knowing much about them.

\section{Exercise}

(I) Part 1. Write all words out on separate, small pieces of paper. (You may still have these from earlier exercises.)

(II) Spread the four headings TOX, LUT, DIB and JEP across a table (or on the floor).

(III) Mix other names in a box.

(IV) Draw one name at a time. Correctly make the TOX and DIB lists and the JEP and LUT trees.

(V) Correct. Practise until completely correct.

(Received for publication May 18, 1982; revision accepted December 8,1982 .) 